Case Report

\title{
Ruptured Multifocal Hepatic Aneurysms in a Woman with Systemic Lupus Erythematosus Successfully Treated with Transcatheter Arterial Embolization: A Case Report and Literature Review
}

\author{
Sz-Iuan Shiu $\mathbb{D}^{1,2}$ Su Ann Yong, ${ }^{3}$ Kuo-Lung Lai, ${ }^{4}$ Chih-Wei Tseng, \\ Chen-Yu Wang, ${ }^{2}$ Bor-Jen Lee $\mathbb{D}^{2}{ }^{2}$ and Yi-Hsing Chen $\mathbb{D}^{4,5}$ \\ ${ }^{1}$ Division of Gastroenterology and Hepatology, Department of Internal Medicine, Taichung Veterans General Hospital, \\ Taichung, Taiwan \\ ${ }^{2}$ Department of Critical Care Medicine, Taichung Veterans General Hospital, Taichung, Taiwan \\ ${ }^{3}$ China Medical University, Taichung, Taiwan \\ ${ }^{4}$ Division of Allergy, Immunology and Rheumatology, Department of Internal Medicine, \\ Taichung Veterans General Hospital, Taichung, Taiwan \\ ${ }^{5}$ Faculty of Medicine, National Yang-Ming University, Taipei, Taiwan \\ Correspondence should be addressed to Yi-Hsing Chen; ysanne@vghtc.gov.tw
}

Received 16 January 2019; Accepted 13 February 2019; Published 26 February 2019

Academic Editor: Hiromasa Ohira

Copyright (c) 2019 Sz-Iuan Shiu et al. This is an open access article distributed under the Creative Commons Attribution License, which permits unrestricted use, distribution, and reproduction in any medium, provided the original work is properly cited.

To present a first reported case of ruptured multifocal hepatic aneurysms in a woman with systemic lupus erythematosus (SLE) who was treated successfully with transcatheter arterial embolization (TAE) in literature, similar cases in the previous English literature were also reviewed and analyzed to summarize the clinical manifestations, management, and outcome in these patients. The data were gathered from the medical record and literature reviews were searched from PudMed. In our review, patients with SLErelated hepatic aneurysms were often middle-aged females. Most of them presented with acute abdominal pain and hypotension. The overall mortality rate was $50 \%$, but it was lower $(12.5 \%)$ in patients who received TAE. Both TAE and surgical intervention are used to treat SLE-related hepatic aneurysms. Our review raised concerns about early detection, diagnosis, and prompt intervention of possible hepatic aneurysm rupture in patients with SLE.

\section{Introduction}

Hepatic aneurysms are rare with an estimated incidence of approximately $0.002 \%$. Very few cases have been reported in patients with systemic lupus erythematosus (SLE) [1] and the pathological mechanism, optimal treatment, and prognosis in this patient population are not clear. We present a case of multifocal hepatic aneurysm rupture in a woman with SLE successfully treated with transcatheter arterial embolization (TAE).

\section{Case Presentation}

A 35-year-old woman presented to the emergency department (ED) complaining of sudden-onset, persistent, moderately severe, left-sided headache with focal left visual field defect followed by right limb clumsiness three hours priorly. She was diagnosed with SLE 11 years previously after developing nephritis, intermittent arthritis, thrombocytopenia, and chronic leg ulcers. She was lupus anticoagulant positive. Treated with monthly cyclophosphamide pulse therapy 
followed by trimonthly injections for the first two and a half years, she remained free of flares on daily maintenance therapy (azathioprine, $50 \mathrm{mg}$; hydroxychloroquine, $200 \mathrm{mg}$; prednisolone, $5 \mathrm{mg}$ (0.05 mg/kg/day); and aspirin, $100 \mathrm{mg})$.

Neurological exam revealed right homonymous hemianopsia without facial palsy but with right-sided hyperesthesia and dysmetria. Brain computed tomography (CT) and magnetic resonance angiography showed acute cerebral infarction in the left posterior cerebral artery territory involving the thalamus and occipital lobe complicated by minimal left temporo-occipital subarachnoid hemorrhage. She was weakly positive for lupus anticoagulant and borderline positive for anticardiolipin antibodies.

Her symptoms gradually improved, but she developed sudden-onset, severe epigastric pain with tachycardia, hypotension, and altered level of consciousness two weeks after hospitalization. Her hemoglobin dropped from 114 to 88 $\mathrm{g} / \mathrm{L}$, and abdominal CT angiography (CTA) showed a massive subcapsular hematoma with contrast extravasation in the left lateral segment of the liver (Figure 1). Emergent angiography showed diffuse hepatic artery aneurysms bilaterally over the liver parenchyma with contrast extravasation from a left hepatic subcapsular hematoma (Figure 2). Diagnosed with hepatic aneurysm rupture, embolization of left proximal hepatic artery with Gelfoam cubes was performed. However, her tachycardia persisted, and her hemoglobin was $66 \mathrm{~g} / \mathrm{L}$ the next day. Follow-up CTA showed a new hematoma over liver segment 7/8. A second superselective TAE with Gelfoam cubes was performed via branches of right hepatic artery after which her vital signs stabilized. Concerned that antiphospholipid antibodies were responsible for the vascular events, rituximab was administered (500 $\mathrm{mg}$ in two consecutive doses two weeks apart). Follow-up abdominal CTA three months later showed resolution of the hepatic aneurysms (Figure 3).

\section{Discussion}

Hepatic aneurysm rupture is rare but life-threatening. SLErelated hepatic aneurysms are even rarer, with only 13 reported cases in the literature [1-8]. Mean patient age at admission was approximately 34 years with a female predominance (71\%) (Table 1). Mean SLE duration was 7.1 years with three-fourths of patients using continuous steroids and only $7 \%$ being hypertensive. Most patients presented with acute abdominal pain (78.6\%) and hypotension (57.1\%). The hepatic aneurysms were on the right lobe in $50 \%$, left lobe in $41.6 \%$, and bilateral lobes in $8.4 \%$. Approximately $83 \%$ of patients had a ruptured aneurysm at presentation. The overall mortality rate was $50 \%$, but it was lower (12.5\%) in patients who received TAE. Lobectomy may be required as rescue therapy in $21.4 \%$ of patients.

The precise mechanism of hepatic aneurysm in SLE is not yet clear. Several hypotheses have been proposed including collagen degeneration, and destruction of smooth muscle and elastic fibers due to vasculitis as well as long-term steroid use [8]. Kurata et al. proposed two different pathogenic mechanisms for aortic aneurysms in lupus patients [9]. In younger patients, systemic vasculitis causes cystic medial

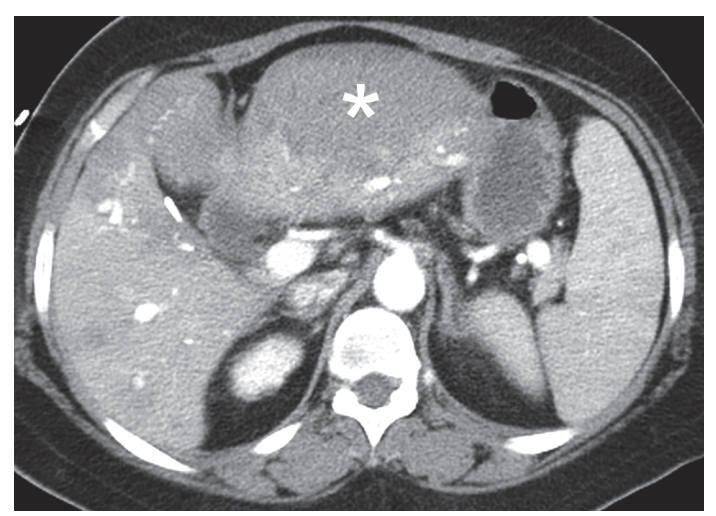

FIGURE 1: The computed tomographic angiography of abdomen of first time revealed subcapsular hematoma (asterisk) with contrast extravasation at left lateral segment of liver.

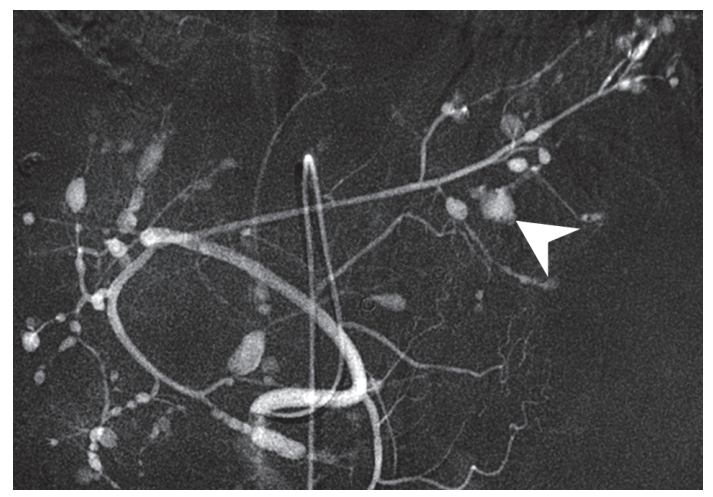

FIGURE 2: The angiography of abdomen revealed diffuse hepatic artery aneurysms over bilateral liver parenchyma with contrast extravasation over branches of left hepatic artery (arrowhead).

degeneration leading to aortic dissection. In older patients on long-term glucocorticoid therapy, aneurysm formation is more likely to be caused by atherosclerosis due to aging. Our patient was relatively young, and the aneurysms resolved with treatment, so vasculitis is more likely to be the cause.

Owing to the large proportion of patients presenting with rupture as the first and only manifestation of hepatic aneurysm, the mortality rate is high, so rapid, appropriate diagnosis and management are crucial. We recommend CTA for diagnosis. Both TAE and surgical intervention are used to treat hepatic aneurysms [1, 10-13]. Choice of treatment modality depends on patient vital signs, aneurysm location, and presence of hepatic collateral circulation. TAE is preferred in patients with a patent portal vein who do not have obstructive jaundice but are a high surgical risk. Surgical intervention with vascular reconstruction is preferred in patients with obstructive jaundice and profound hypovolemic shock despite rapid resuscitation. In our case, prophylactic embolization of the main hepatic artery was performed during the second TAE to reduce intra-aneurysmal pressure, which may have contributed to shrinkage and disappearance of the aneurysms found at her three-month follow-up visit. 


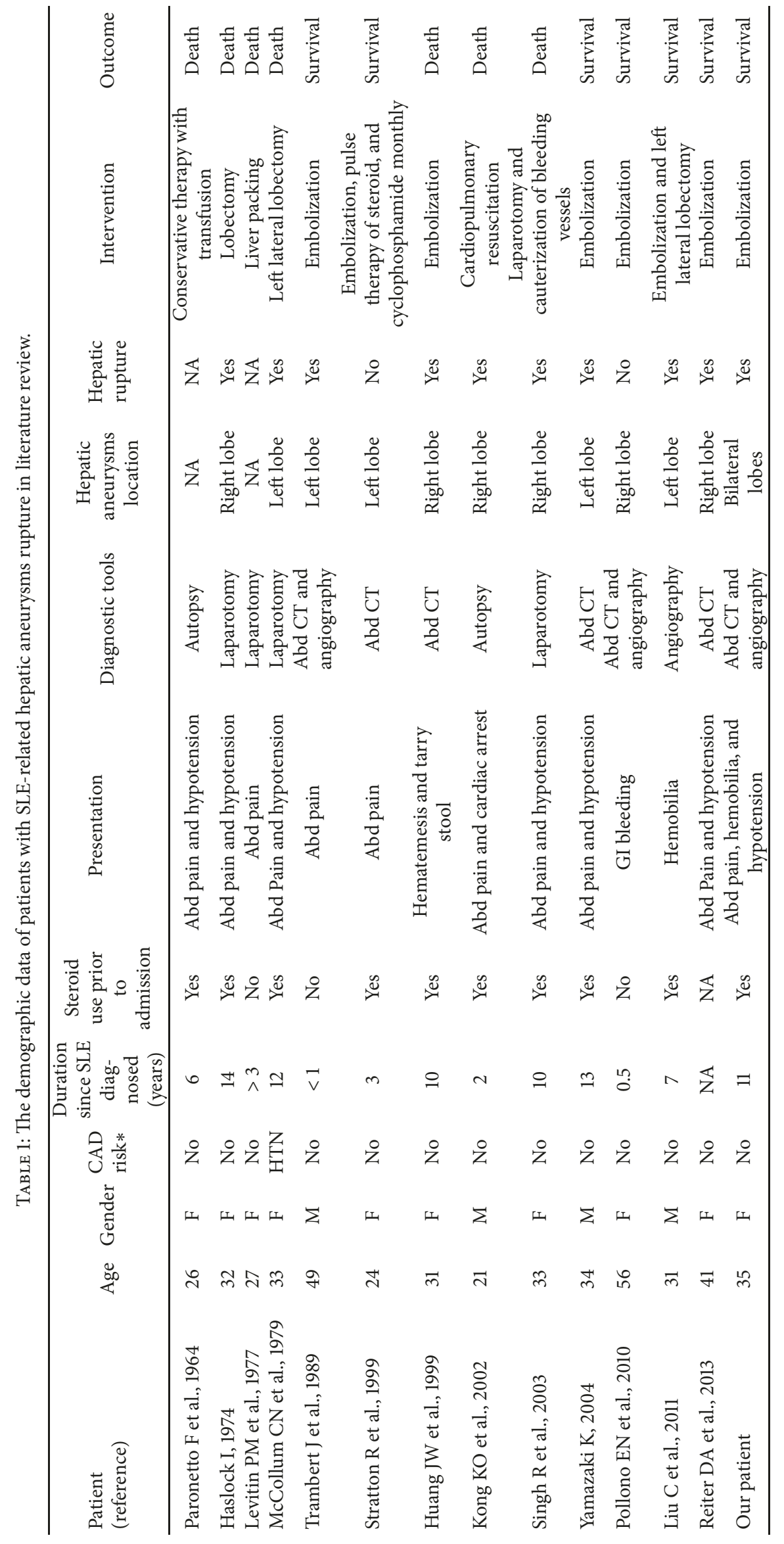




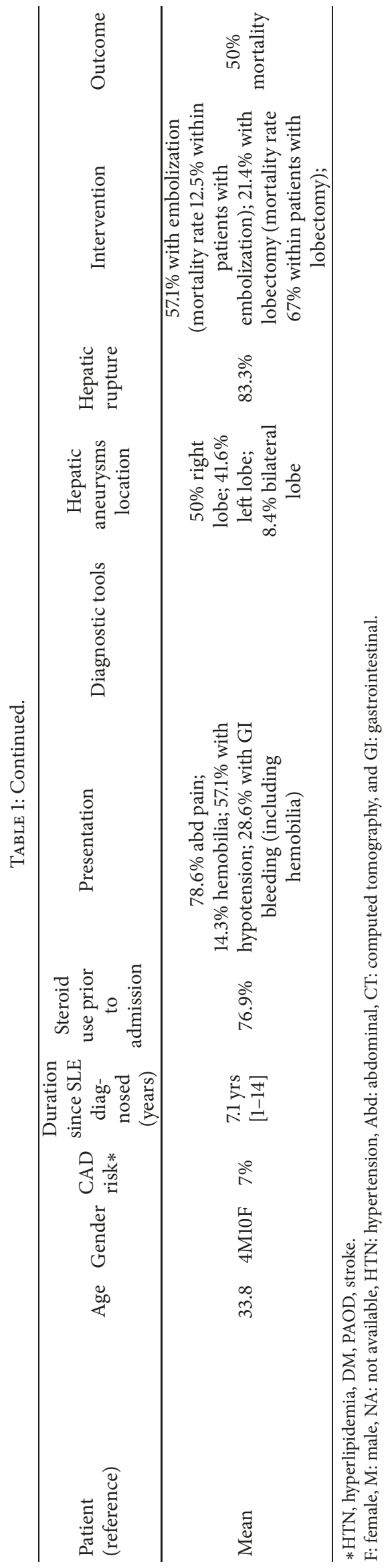




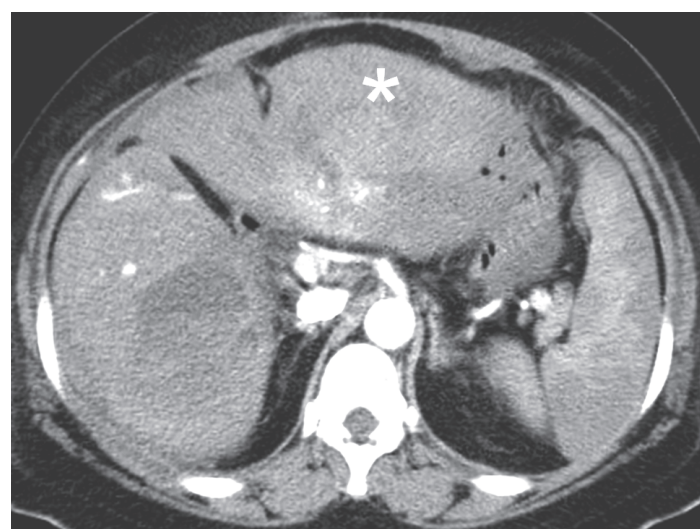

FIGURE 3: The computed tomographic angiography of abdomen 3 months later showed complete resolution of subscapular hematoma (asterisk). A residual hematoma over right lobe of liver was noted but without any clinical manifestation.

B cell depletion with the anti-CD20 monoclonal rituximab has been found to be effective in patients with SLE in several open-label cohort studies [14] and rituximab has also been suggested in refractory SLE [15]. Under the concern that hepatic aneurysm might be related to refractory SLE, we prescribed rituximab as induction and maintenance therapy for residual hepatic aneurysm after embolization. No residual hepatic aneurysm was discovered at her three-month followup visit.

To our knowledge, this is the first reported case of bilateral multifocal hepatic aneurysm rupture in a young woman with SLE successfully treated with TAE. It emphasizes the need for rapid diagnosis and treatment to achieve a successful clinical outcome in patients with hepatic artery aneurysm.

\section{Conflicts of Interest}

The authors declare that they have no conflicts of interest.

\section{Authors' Contributions}

Sz-Iuan Shiu and Su Ann Yong contributed equally to this work.

\section{Acknowledgments}

Evidence-based Practice and Policymaking Committee, Taichung Veterans General Hospital, Taichung, Taiwan, is acknowledged.

\section{References}

[1] D. A. Reiter, A. M. Fischman, and B. D. Shy, "Hepatic artery pseudoaneurysm rupture: a case report and review of the literature," The Journal of Emergency Medicine, vol. 44, no. 1, pp. 100-103, 2013.

[2] F. Paronetto, L. Deppisch, and L. R. Tuchman, "Lupus erythematosus with fatal hemorrhage into the liver and lesions resembling those of periarteritis nodosa and malignant hypertension. Immunocytochemical observations," American Journal of Medicine, vol. 36, no. 6, pp. 948-955, 1964.

[3] P. M. Levitin, D. Sweet, and C. M. Brunner, "Spontaneous rupture of the liver. an unusual complication of SLE," Arthritis \& Rheumatology, vol. 20, no. 2, pp. 748-750, 1977.

[4] C. N. McCollum, M. E. Sloan, A. M. Davison, and G. R. Giles, "Ruptured hepatic aneurysm in systemic lupus erythematosus," Annals of the Rheumatic Diseases, vol. 38, no. 4, pp. 396-398, 1979.

[5] J. Trambert, E. Reinitz, and S. Buchbinder, "Ruptured hepatic artery aneurysms in a patient with systemic lupus erythematosus: case report," CardioVascular and Interventional Radiology, vol. 12, no. 1, pp. 32-34, 1989.

[6] E. N. Pollono, D. C. Madoff, S. C. Spence, and M. E. SuarezAlmazor, "Multiple hepatic artery aneurysms in a patient with systemic lupus erythematosus," Lupus, vol. 19, no. 1, pp. 93-95, 2010.

[7] R. Singh, Y. Menon, H. McGrath, and L. R. Espinoza, "Acute abdomen due to spontaneous organ rupture in systemic lupus erythematosus," The American Journal of Medicine, vol. 114, no. 2, pp. 160-161, 2003.

[8] J.-W. Huang, M.-S. Wu, T.-S. Chu, and T.-J. Tsai, "Hepatic aneurysm and portal vein thrombosis in a patient with lupus erythematosus on dialysis," Nephrology Dialysis Transplantation , vol. 14, no. 3, pp. 753-756, 1999.

[9] A. Kurata, T. Kawakami, J. Sato, A. Sakamoto, T. Muramatsu, and K. Nakabayashi, "Aortic aneurysms in systemic lupus erythematosus: a meta-analysis of 35 cases in the literature and two different pathogeneses," Cardiovascular Pathology, vol. 20, no. 1, pp. e1-e7, 2011.

[10] D. P. Nathan, G. J. Wang, E. Y. Woo, R. M. Fairman, and B. M. Jackson, "Open and endovascular repair of hepatic artery aneurysm: two case reports and review of the literature," Vascular, vol. 19, no. 1, pp. 42-46, 2011.

[11] A. Gabelmann, J. Görich, and E. M. Merkle, "Endovascular treatment of visceral artery aneurysms," Journal of Endovascular Therapy, vol. 9, no. 1, pp. 38-47, 2002.

[12] N. Tulsyan, V. S. Kashyap, R. K. Greenberg et al., "The endovascular management of visceral artery aneurysms and pseudoaneurysms," Journal of Vascular Surgery, vol. 45, no. 2, pp. 276-283, 2007.

[13] S. S. Saltzberg, T. S. Maldonado, P. J. Lamparello et al., "Is endovascular therapy the preferred treatment for all visceral artery aneurysms?" Annals of Vascular Surgery, vol. 19, no. 4, pp. 507-515, 2005.

[14] J. Furtado and D. A. Isenberg, "B cell elimination in systemic lupus erythematosus," Clinical Immunology, vol. 146, no. 2, pp. 90-103, 2013.

[15] B. Duxbury, C. Combescure, and C. Chizzolini, "Rituximab in systemic lupus erythematosus: an updated systematic review and meta-analysis," Lupus, vol. 22, no. 14, pp. 1489-1503, 2013. 


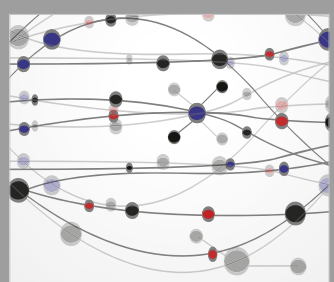

The Scientific World Journal
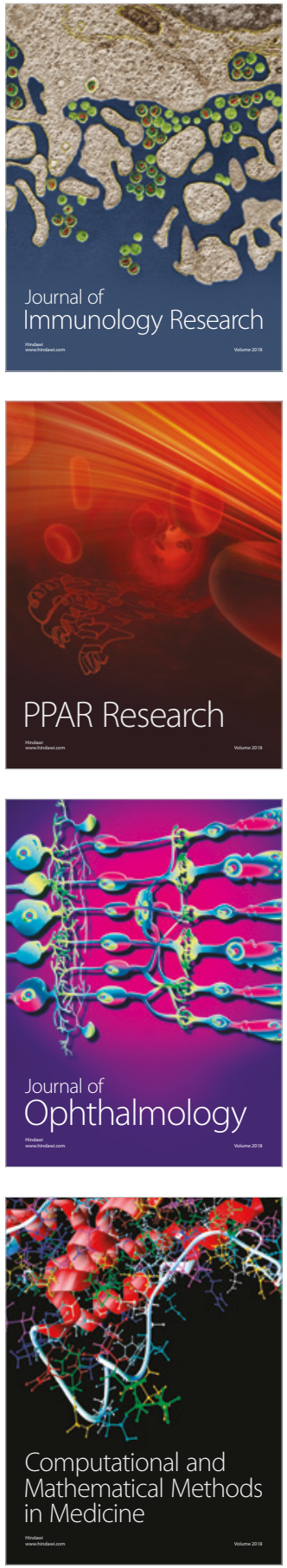

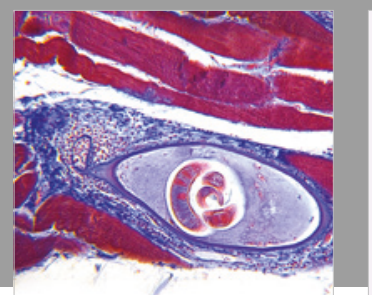

Gastroenterology Research and Practice

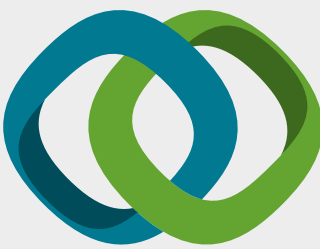

\section{Hindawi}

Submit your manuscripts at

www.hindawi.com
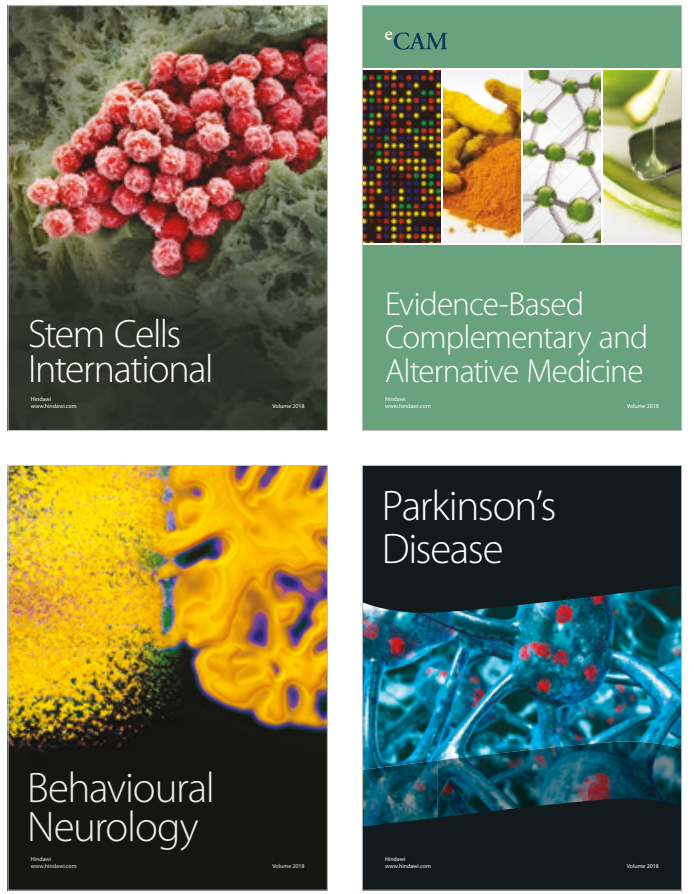

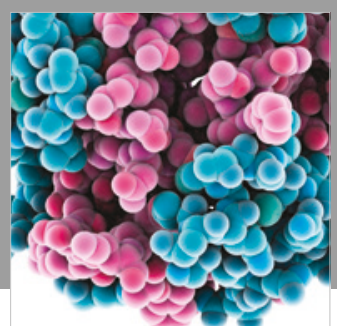

ournal of

Diabetes Research

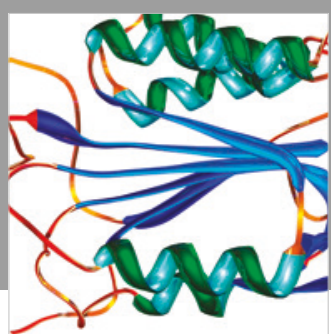

Disease Markers
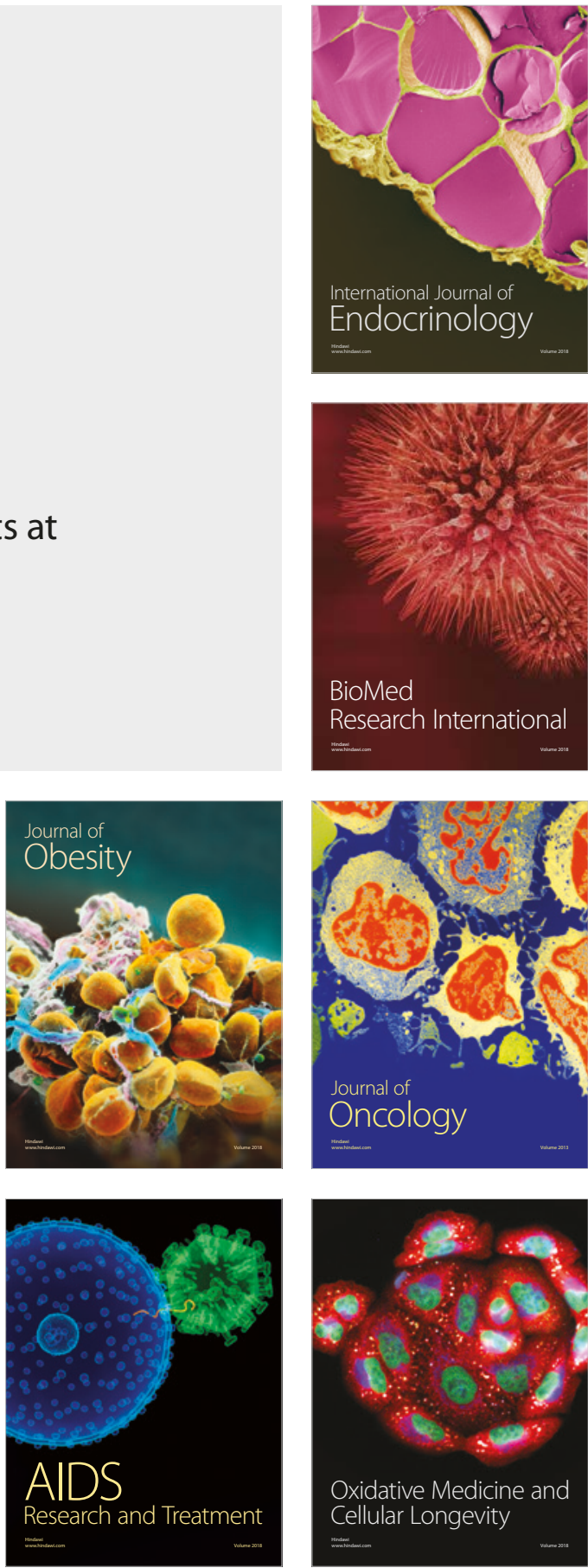